\title{
Behaviour and development in 24-month-old moderately preterm toddlers
}

\author{
Marjanneke de Jong, ${ }_{1}^{1}$ Marjolein Verhoeven, ${ }_{1}^{1}$ Carole A Lasham, ${ }^{2}$ \\ Clemens B Meijssen, ${ }^{3}$ Anneloes L van Baar ${ }^{1}$
}

${ }^{1}$ Department of Child and Adolescent Studies, Utrecht University, Utrecht, The Netherlands

${ }^{2}$ Department of Pediatrics, Tergooi Hospital, Blaricum The Netherlands ${ }^{3}$ Department of Pediatrics, Meander Medical Centre, Amersfoort, The Netherlands

\section{Correspondence to}

Marjanneke de Jong, Department of Child and Adolescent Studies, Utrecht University, P.O. Box 80.140 3508 TC Utrecht,

The Netherlands; M.deJong@uu.nl

Received 20 June 2014 Revised 19 December 2014 Accepted 29 December 2014 Published Online First 14 January 2015

\section{CrossMark}

To cite: de Jong $\mathrm{M}_{\text {, }}$ Verhoeven M, Lasham CA, et al. Arch Dis Child 2015;100:548-553.

\section{ABSTRACT \\ Objective Moderately preterm children (gestational} age 32-36+6 weeks) are at risk of cognitive and behaviour problems at school age. The aim of this study was to investigate if these problems are already present at the age of 2 years.

Study design Developmental outcome was assessed at 24-months (corrected age) with the Bayley-III-NL in 116 moderately preterm $(M=34.66 \pm 1.35$ weeks gestation) and 99 term born children $(M=39.45$ \pm 0.98 weeks gestation). Behaviour problems were assessed with the Child Behaviour Checklist.

Results With age corrected for prematurity, moderately preterm children scored below term peers on Receptive Communication skills $(11.05 \pm 2.58$ vs $12.02 \pm 2.74$, $\mathrm{p}=0.02$ ). Without correcting age for prematurity, moderately preterm children scored below term born peers on Cognition (8.97 \pm 2.11 vs $10.68 \pm 2.35$, $\mathrm{p}<0.001)$, Fine Motor $(10.33 \pm 2.15$ vs $11.96 \pm 2.15$, $p<0.001)$, Gross Motor $(8.47 \pm 2.55$ vs $9.39 \pm 2.80$, $\mathrm{p}=0.05)$, Receptive Communication $(10.09 \pm 2.48$ vs $12.02 \pm 2.74, p<0.001)$ and Expressive Communication $(10.33 \pm 2.43$ vs $11.49 \pm 2.51, p=0.005)$ skills. Compared with term peers, more moderately preterm children showed a (mild) delay (ie, scaled score $<7$ ) in gross motor skills with age uncorrected for prematurity $(20.7 \%$ vs $11.2 \%, p=0.04)$. Moderately preterm children had more internalising behaviour problems than term children (44.76 \pm 8.94 vs $41.54 \pm 8.56, p=0.03$ ). No group differences were found in percentages of (sub)clinical scores.

Conclusions At the age of 2 years, uncorrected for prematurity, differences in cognition, communication, and motor development were present in moderately preterm children compared with term born peers. After correcting age for prematurity, a difference was only found for receptive communication skills. In addition, moderately preterm children show more internalising behaviour problems.

\section{INTRODUCTION}

Worldwide, $11.1 \%$ of all pregnancies end in preterm delivery before 37 weeks of gestation, of which $84 \%$ are born moderately preterm after a gestational age (GA) between 32 to 36 weeks and 6 days. $^{1}$ Although to a lesser extent than very and extreme preterm children, moderately preterm children still have more neonatal complications than term born children, such as respiratory distress, hypoglycaemia and feeding problems. ${ }^{2}$ Long-term follow-up studies have shown that these children are at increased risk of cognitive, school and behaviour problems at (pre)school age. ${ }^{3}$ Little is known

\section{What is already known on this topic}

Moderately preterm children are at risk for cognitive, school and behaviour problems.

- Little is known about moderately preterm born children at toddler age, especially with respect to standardised behavioural outcomes.

- Most previous studies studied American populations, less is known about the development of European moderately preterm children.

\section{What this study adds}

- Using chronological age, moderately preterm toddlers scored lower than term born peers on cognition, communication and motor skills.

- Even after correcting age for prematurity, moderately preterm 24-month-olds showed lower receptive communication scores (Bayley-III-NL).

- Moderately preterm children have more internalising behaviour problems (Child Behavior Checklist) already at toddler age.

about developmental and behavioural outcomes in moderately preterm children in their first 2 years of life, even though this may be a period during which the first signs of developmental delay appear. The aims of the current study are to investigate developmental and behavioural outcomes of 24-month-old moderately preterm children compared with term born peers.

Few studies have compared developmental outcomes (ie, cognitive, motor and/or communication skills) of moderately preterm with term born toddlers at this early age, using developmental tests. These studies showed inconsistent results, with some studies finding poorer outcomes for moderately preterm children, ${ }^{4-9}$ while others found no

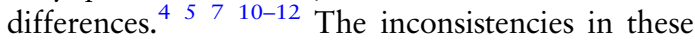
findings are most likely a result of the use of age correction for prematurity or not. Age correction is infrequently used in moderately preterm children, especially in the late preterm subgroup (ie, GA of 35-36 weeks). Most studies that found group differences did not correct age for prematurity, ${ }^{5-8}$ while the studies that found no differences did correct the ages. ${ }^{5}{ }^{7}$ 10-12 Two studies did find 
differences with scores based on corrected age. ${ }^{4}{ }^{9}$ Hillemeier et $a l^{4}$ found that moderately preterm children had double the risk for scoring in the lowest $10 \%$ at 9 months of age, but by 24 months of age there was no increased risk in the moderately preterm children. Voigt et $a l^{9}$ found a difference, with lower scores for moderately preterm children, with a medium effect size at 24 months of age. In the current study, differences between moderately preterm and term born toddlers will be determined for corrected as well as uncorrected age.

Despite growing evidence that moderately preterm children show more problem behaviour at (pre)school age, ${ }^{3}{ }^{13-15}$ no studies have been published that have determined the prevalence of behavioural problems in this group at toddler age using standardised measures such as the Child Behaviour Checklist (CBCL

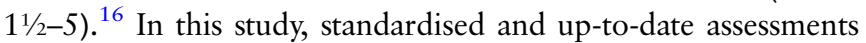
were performed in moderately preterm and term born children at 2 years of age in order to compare functioning of both groups.

\section{METHOD}

This study is part of an ongoing longitudinal project on the development of Dutch moderately preterm children, the STAP Project (ie, Study on Attention of Preterm children). Parents of moderately preterm (32-36 weeks' gestation) and term born ( $>37$ weeks' gestation) children were invited by letter by their paediatrician or midwife to participate in the study when their child was 10 months old. Children were born between March 2010 and April 2011 in nine hospitals in and around Utrecht. For both groups, exclusion criteria were dysmaturity (birth weight below the 10th centile according to Dutch reference curves ${ }^{17}$ ), multiple birth, severe congenital malformations, antenatal alcohol or drug abuse by the mother, and chronic antenatal use of psychiatric drugs by the mother. We also excluded children admitted to a tertiary neonatal intensive care unit (NICU), according to the admission criteria of the official guidelines from the Dutch Society for Obstetrics and Gynecology and the Dutch Pediatric Association, as this might be a specific subgroup. In the Dutch population in 2012, NICU treatment was needed in $29 \%$ of the children born after 32-33 weeks and only in 7\% of infants born after 34-36 weeks. ${ }^{18}$

Informed consent was given by the parents. The children received a gift after the visit and parents received travel expenses.

\section{Measures}

Developmental level

At 24 months of age, corrected for prematurity, a trained examiner performed the Dutch version of the Bayley-III, the Bayley-III-NL ${ }^{19}$ to assess the developmental level of the children.

The Bayley-III-NL consists of five subtests: Cognition, Fine Motor, Gross Motor, Receptive Communication and Expressive Communication. Scaled scores based on Dutch norms were used, which vary between 1 and 19 with a mean of 10 and a SD of 3. Scores of 7-13 are considered normal; a score below 7 indicates a (mild) developmental delay, ${ }^{20}$ and is therefore seen as a clinically relevant score in this study. The reliability and validity of the Bayley-III-NL is good. ${ }^{20}$

\section{Behaviour}

Prior to the visit to the test location, mothers completed the

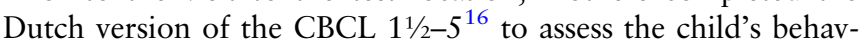
iour problems. The CBCL $1 \frac{1 / 2-5}{2}$ consists of 100 items with descriptions of behaviour problems for which the mother indicates to what extent this fits the child now or in the past 2 months ('not/never', 'somewhat/sometimes' and 'very/often'). The T-scores of the total problem scale, as well as the T-scores on the two broadband scales (ie, internalising and externalising behaviour) and the seven subscales (ie, emotional reactivity, anxious/depressed behaviour, somatic complaints, withdrawn behaviour, sleep problems, attention problems and aggressive behaviour) were used. For total problems and the broadband scales, T-scores below 60 are considered normal, between 60 and 64 is seen as borderline clinical, and 64 or higher as clinical scores. ${ }^{16}$ For the subscales, scores below 65 are considered normal, between 65 and 70 borderline clinical, and 70 or higher as clinical scores. ${ }^{16}$ Subclinical and clinical scores are considered as clinically relevant scores in this study. The reliability and validity of the CBCL $1 \frac{1 / 2-5}{\text { is good. }}{ }^{16}$

\section{Neonatal and background characteristics}

Neonatal characteristics regarding hypoglycaemia, phototherapy, oxygen requirements, and length of hospital stay were based upon the discharge letters in the hospital files. Background characteristics, such as maternal education level, maternal age at birth and ethnic origin of the child, were provided by the parents using a short questionnaire.

\section{Data analysis}

Group differences in scores were investigated using (Multivariate) Analyses of Covariance ((M)ANCOVAs). Group differences in percentages of clinically relevant scores were investigated with logistic regression analyses. Analyses were adjusted for background characteristics that differed between the groups. (Multivariate) Analyses of Variance and Pearson's correlations were used to explore the relationship between neonatal characteristics and outcome measures within the moderately preterm born group.

Power analysis using $\mathrm{G}^{*}$ Power showed that to test a group difference with a medium effect size (Cohen's $\mathrm{f} 2=0.15$ ), a significance level of $\alpha=0.05$ and a power of 0.80 , using a MANCOVA with seven dependent variables, a sample of in total 104 was needed, with 52 children in each group. ${ }^{21}$ Partial $\eta^{2}$ was used as effect size estimate, with 0.01 indicating a small effect size, 0.06 a medium effect size, and 0.13 and above a large effect size. ${ }^{22}$

\section{RESULTS}

Of the 333 parents of moderately preterm children that were eligible for this study in the participating hospitals, 123 consented (37\%). The participating moderately preterm children did not differ from non-participants in GA, birth weight, number of days in hospital, additional oxygen requirement, phototherapy requirement, gender and percentage of first-born children. A slightly higher incidence of hypoglycaemia was observed in non-participants $\left(11.2 \%\right.$ vs $4.9 \%, \chi^{2}=3.76$, $\mathrm{p}=0.05$ ). For the 457 eligible term born children, parents of 103 children $(23 \%)$ consented to participate. The participating term born children did not differ from non-participants in gender, GA, birth weight, number of days in hospital, additional oxygen requirement, phototherapy requirement, hypoglycaemia and percentage of first-borns. The distribution of GA in weeks of the preterm and term born sample did not differ significantly from that in the general Dutch population. ${ }^{18}$

Complete data was available for 116 (94\%) moderately preterm children and for 99 (96\%) term born children. Sample characteristics are shown in table 1 . The groups differed in maternal education level and maternal age at birth, with more low and medium educated mothers and younger mothers in the 
Table 1 Neonatal and demographic characteristics of the participants

\begin{tabular}{|c|c|c|c|}
\hline & $\begin{array}{l}\text { Term born } \\
\text { GA 37-41 weeks } \\
(n=99)\end{array}$ & $\begin{array}{l}\text { Moderately preterm } \\
\text { GA 32-36 weeks } \\
(n=116)\end{array}$ & \\
\hline \multicolumn{4}{|l|}{ Neonatal characteristics } \\
\hline \multicolumn{4}{|l|}{ GA in weeks } \\
\hline Mean (SD) & $39.45(0.98)$ & $34.66(1.35)$ & \\
\hline 32 weeks (\%) & & $10.3 \%$ & \\
\hline 33 weeks (\%) & & $11.2 \%$ & \\
\hline 34 weeks (\%) & & $17.2 \%$ & \\
\hline 35 weeks (\%) & & $24.1 \%$ & \\
\hline 36 weeks (\%) & & $37.1 \%$ & \\
\hline 37 weeks (\%) & $4.0 \%$ & & \\
\hline 38 weeks (\%) & $11.1 \%$ & & \\
\hline 39 weeks (\%) & $32.3 \%$ & & \\
\hline 40 weeks (\%) & $40.4 \%$ & & \\
\hline 41 weeks (\%) & $12.1 \%$ & & \\
\hline \multicolumn{4}{|l|}{ Birth weight in grams } \\
\hline Mean (SD) & $3575(460)$ & $2575(508)$ & \\
\hline Range & $2795-5330$ & $1420-3850$ & \\
\hline \multicolumn{4}{|l|}{ Days in hospital } \\
\hline Mean (SD) & $0.42(1.01)$ & $12.00(9.84)$ & \\
\hline Range & $0-6$ & $1-42$ & \\
\hline Need for oxygen* $(\%)$ & $0 \%$ & $22.4 \%$ & \\
\hline Phototherapy (\%) & $0 \%$ & $35.3 \%$ & \\
\hline Hypoglycaemia (\%) & $0 \%$ & $5.2 \%$ & \\
\hline Demographic characteristics & & & $p$ Valuet \\
\hline \multicolumn{4}{|l|}{ Age in months‡ } \\
\hline Mean (SD) & $23.71(0.52)$ & $23.60(0.63)$ & 0.20 \\
\hline Range & $23-25$ & $23-27$ & \\
\hline Gender (\% boys) & $45.5 \%$ & $57.8 \%$ & 0.07 \\
\hline First-born (\%) & $51.5 \%$ & $62.9 \%$ & 0.09 \\
\hline Ethnic origin (\% Dutch) & $95.9 \%$ & $96.5 \%$ & 0.42 \\
\hline \multicolumn{4}{|l|}{ Maternal age at birth (years) } \\
\hline Mean (SD) & $32.52(4.20)$ & $31.04(4.43)$ & 0.01 \\
\hline Range & $20-43$ & $21-41$ & \\
\hline \multicolumn{4}{|l|}{ Maternal educational level } \\
\hline Low§ & $3.0 \%$ & $7.8 \%$ & \\
\hline Mediumๆ & $12.1 \%$ & $35.3 \%$ & $<0.001$ \\
\hline High** $^{* *}$ & $84.8 \%$ & $56.9 \%$ & \\
\hline
\end{tabular}

moderately preterm group. Therefore, we controlled for maternal education level and maternal age at birth in all analyses concerning group differences.

\section{Developmental outcomes}

The average scaled scores and the percentage of children with a (mild) developmental delay, which reflects a score below 7 on the subtests of the Bayley-III-NL are presented per group in tables 2 and 3, respectively. For the moderately preterm group, the results are presented separately for corrected and uncorrected age (for prematurity).

Group differences using age corrected for prematurity A MANCOVA of the five subtests of the Bayley-III-NL showed no overall difference between moderately preterm and term born children on the scaled scores after correcting age for prematurity and adjusting for maternal education level and maternal age at birth. However, moderately preterm children scored significantly lower than full term peers regarding Receptive Communication with a small effect size. No group differences were found in the percentages of children with a mild developmental delay, see table 3 .

Group differences using uncorrected, chronological age When age was not corrected for prematurity, the MANCOVA showed a significant difference between moderately preterm and term born children. Moderately preterm children scored significantly below term born children on all subtests, that is, Cognition, Fine Motor skills, Gross Motor skills, Receptive Communication and Expressive Communication with small to 
Table 2 Developmental outcomes of the children on the Bayley-III-NL

\begin{tabular}{|c|c|c|c|c|c|c|c|}
\hline & \multirow{2}{*}{$\begin{array}{l}\mathrm{FT} \\
\mathrm{N}=98 \\
\text { Mean (SD) }\end{array}$} & \multicolumn{3}{|c|}{$\begin{array}{l}\text { MPT-corrected age } \\
\mathrm{N}=116\end{array}$} & \multicolumn{3}{|c|}{$\begin{array}{l}\text { MPT_-uncorrected age } \\
\mathrm{N}=116\end{array}$} \\
\hline & & Mean (SD) & p Value* & Effect sizet & Mean (SD) & p Value* & Effect size \\
\hline Cognition & $10.68(2.35)$ & $10.01(2.27)$ & 0.26 & 0.01 & $8.97(2.11)$ & $<0.001$ & 0.09 \\
\hline Fine motor & $11.96(2.15)$ & $11.34(2.06)$ & 0.09 & 0.01 & $10.33(2.15)$ & $<0.001$ & 0.11 \\
\hline Gross motor & $9.39(2.80)$ & $9.26(2.78)$ & 0.88 & 0.00 & $8.47(2.55)$ & 0.05 & 0.02 \\
\hline Receptive communication & $12.02(2.74)$ & $11.05(2.58)$ & 0.02 & 0.03 & $10.09(2.48)$ & $<0.001$ & 0.11 \\
\hline Expressive communication & $11.49(2.51)$ & $11.15(2.54)$ & 0.59 & 0.001 & $10.33(2.43)$ & 0.005 & 0.04 \\
\hline
\end{tabular}

MANCOVA results for corrected age: Wilks' $\Lambda=0.96, F_{5,206}=1.80, p=0.11$, partial $\eta^{2}=0.04$

MANCOVA results for uncorrected age: Wilks' $\Lambda=0.83, \mathrm{~F}_{5,206}=8.26, \mathrm{p}<0.001$, partial $\eta^{2}=0.17$.

${ }^{*}$ Compared with full term group. †Partial $\eta^{2}$.

FT, full term, GA 37-41 weeks; GA, gestational age; MANCOVA, multivariate analysis of covariance; MPT, moderately preterm, GA 32-36 weeks; analyses are adjusted for maternal education level and maternal age at birth.

medium effect sizes. A higher percentage of moderately preterm children showed a (mild) developmental delay regarding gross motor skills (see table 3). No group differences were found for the other subtests.

\section{Behavioural outcomes}

The CBCL T-scores are presented in table 4. Moderately preterm children did not differ from term children in total problem scores. A MANCOVA with the T-scores of the internalising and externalising scale also showed no significant group difference. However, moderately preterm children did have significantly higher internalising behaviour scores. No differences were found in externalising behaviour problems. A MANCOVA with the T-scores of the seven subscales of the CBCL showed no group differences. No group differences were found in the percentages of children with (sub)clinical scores, see table 4 .

\section{Neonatal characteristics in relation to outcome measures within the moderately preterm group}

The analyses described above were repeated comparing only late preterm born children (ie, GA 34-36 weeks, $n=91$ ) with the term born group. All results remained the same (data not presented).

Within the moderately preterm group, GA (as continuous variable), birth weight and days in hospital were not related to developmental outcomes (using chronological age and corrected age) or behavioural outcomes; Pearson's correlations between these neonatal characteristics and the Bayley-III or CBCL scores were not significant and varied between 0.001 and 0.17 , except for the correlation between birth weight and the sleep problems subscale of the CBCL that was significant with a small effect size $(r=0.19, \quad p=0.05)$. No differences on behavioural and developmental outcomes were found between children who required additional oxygen and those who did not. Also, no differences were found between the 41 children who received phototherapy and those who did not, and between the 6 children who had hypoglycaemia and those who had normoglycaemia.

\section{DISCUSSION}

This study on outcome of moderately preterm children at 2 years of age showed that using uncorrected, chronological age, resulted in lower scores for cognitive, motor and communication skills, compared with scores of term born children. Even for the (large) subgroup of only late preterm children these results were found. This is in line with previous studies that used uncorrected age. ${ }^{578}$ When we did correct age for prematurity, the group differences generally were not statistically significant, although, overall, preterm children had lower scores than their term born peers. Moderately preterm children did not differ from term born peers in cognitive and motor skills when age-corrected scores were analysed, which is in accordance with some previous studies at infant and toddler age. $^{4} 57$ 10-12 However, even after correcting age for prematurity, a significant difference was found, showing that moderately preterm children scored somewhat below term born peers on receptive communication skills. It is difficult to compare these results with previous studies, as in most previous studies regarding prematurely born toddlers, ${ }^{4}{ }^{6-11}$ language skills were not assessed by a separate subtest, except for the study by Morag et $a l^{5}$, that found no difference between moderately preterm and term born children after correcting age for prematurity.

Little consensus exists whether correcting age for prematurity is needed in moderately preterm children and if so, until what

Table 3 Percentage of children with a (mild) developmental delay (ie, scaled score $<7$ ) on the Bayley-III-NL

\begin{tabular}{|c|c|c|c|c|c|c|c|c|c|}
\hline & \multirow[b]{2}{*}{ FT } & \multicolumn{4}{|c|}{ MPT-corrected age } & \multicolumn{4}{|c|}{ MPT-uncorrected age } \\
\hline & & & OR & $(95 \% \mathrm{Cl})$ & p Value* & & OR & $(95 \% \mathrm{Cl})$ & p Value* \\
\hline Cognition & $3.1 \%$ & $4.3 \%$ & 0.89 & (0.19 to 4.15$)$ & 0.89 & $8.6 \%$ & 2.19 & (0.56 to 8.63$)$ & 0.26 \\
\hline Fine motor & $2.0 \%$ & $0.9 \%$ & 0.48 & (0.04 to 6.36$)$ & 0.57 & $5.2 \%$ & 2.13 & (0.40 to 11.44$)$ & 0.38 \\
\hline Gross motor & $11.2 \%$ & $15.5 \%$ & 1.61 & (0.69 to 3.73 ) & 0.27 & $20.7 \%$ & 2.30 & (1.03 to 5.13$)$ & 0.04 \\
\hline Receptive communication & $2.0 \%$ & $4.3 \%$ & 2.07 & (0.37 to 11.56$)$ & 0.41 & $6.9 \%$ & 3.52 & (0.69 to 17.82$)$ & 0.13 \\
\hline Expressive communication & $6.1 \%$ & $4.3 \%$ & 0.48 & (0.13 to 1.75$)$ & 0.26 & $7.8 \%$ & 1.03 & (0.33 to 3.17$)$ & 0.96 \\
\hline
\end{tabular}

*Compared with full term group; analyses are adjusted for maternal education level and maternal age at birth.

FT, full term, GA 37-41 weeks; GA, gestational age; MPT, moderately preterm, GA 32-36 weeks. 
Table 4 Behavioural outcomes of the children

\begin{tabular}{|c|c|c|c|c|c|c|c|c|c|}
\hline & \multicolumn{4}{|l|}{$\begin{array}{l}\text { T score } \\
\text { Mean (SD) }\end{array}$} & \multicolumn{5}{|c|}{$\%$ (sub)clinical } \\
\hline & $\begin{array}{l}\mathrm{FT} \\
\mathrm{N}=94\end{array}$ & $\begin{array}{l}\text { MPT } \\
N=111\end{array}$ & p Value & Effect size* & FT (\%) & МPT(\%) & OR & $(95 \% \mathrm{Cl})$ & p Value \\
\hline Total problems & $43.34(8.56)$ & $46.06(7.87)$ & 0.08 & 0.02 & 3.2 & 5.4 & 1.37 & (0.31 to 6.02$)$ & 0.68 \\
\hline Internalising problems & $41.54(8.56)$ & $44.76(8.94)$ & 0.03 & 0.03 & 1.1 & 5.4 & 3.70 & (0.41 to 33.09$)$ & 0.24 \\
\hline Externalising problems & $46.80(8.68)$ & $48.80(8.11)$ & 0.24 & 0.01 & 4.3 & 9.0 & 1.88 & (0.54 to 6.54$)$ & 0.32 \\
\hline \multicolumn{10}{|l|}{ Subscales } \\
\hline Emotionally reactive & $51.43(2.98)$ & $52.60(4.59)$ & 0.12 & 0.01 & 1.1 & 4.5 & 3.70 & (0.40 to 34.22$)$ & 0.25 \\
\hline Anxious/ depressed & $50.46(1.55)$ & $50.74(1.88)$ & 0.53 & 0.002 & 0 & 0 & - & - & - \\
\hline Somatic complaints & $52.15(4.23)$ & $53.68(5.49)$ & 0.18 & 0.01 & 3.2 & 10.8 & 2.26 & (0.58 to 8.83$)$ & 0.24 \\
\hline Withdrawn & 51.87 (3.34) & $52.23(3.51)$ & 0.70 & 0.001 & 1.1 & 0.9 & 0.76 & (0.04 to 15.14$)$ & 0.86 \\
\hline Sleep problems & 51.99 (4.09) & $52.13(3.89)$ & 0.89 & 0.00 & 2.1 & 1.8 & 0.53 & (0.06 to 4.43 ) & 0.55 \\
\hline Attention problems & $52.68(4.58)$ & $53.46(5.26)$ & 0.88 & 0.00 & 4.3 & 6.3 & 1.06 & (0.28 to 4.04$)$ & 0.93 \\
\hline Aggressive behaviour & $52.28(3.58)$ & $52.86(4.60)$ & 0.35 & 0.004 & 0 & 3.6 & - & - & - \\
\hline
\end{tabular}

age. This study showed that correcting age for prematurity influences the findings, even in the subgroup of late preterm children. It is, however, as yet unknown which score (ie, corrected or uncorrected for prematurity) is most predictive for later functioning. A few studies examined the predictive value of corrected and uncorrected scores in very preterm children with inconsistent results. $^{23-25}$ In two studies, ${ }^{23}$ no difference in predictive validity of corrected and uncorrected scores was found. In contrast, Rickards et $a l^{25}$ reported that the corrected scores were most predictive of later outcomes. Further research is needed to investigate the predictive value of corrected and uncorrected scores in moderately preterm children.

The percentage of children showing a developmental delay was not higher in the moderately preterm group for most of the subtests, except for gross motor skills when age was uncorrected for prematurity. For both groups, the percentage of children showing a developmental delay was lower than expected for all subtests, except for gross motor skills. In addition, the mean scores of all subtests, except gross motor skills, were higher than the normative means. This might be explained by the fact that a sample of relatively highly educated mothers participated in this study and term healthy children of higher educated mothers have been found to show higher Bayley-III scores. ${ }^{20}$

Moderately preterm 24-month-olds were found to show somewhat more internalising behaviour problems than full term peers. No differences between levels of externalising behaviours were found. Despite these higher mean levels of problem behaviour, moderately preterm children did not show a higher risk of (sub)clinical scores than term born children. Our findings are partly in accordance with the study of Potijk et al, ${ }^{14}$ who found significantly higher problem scores for 4-year-old moderately preterm children on all scales of the CBCL. It might be that the behaviour problems of moderately preterm children increase with age, as several studies showed at preschool ${ }^{13}{ }^{14}$ and school age $^{13} \quad 1526$ that moderately preterm children had a higher risk of subclinical scores for total behaviour problems, ${ }^{13}{ }^{14}$ internalising behaviour, ${ }^{15} 26$ externalising behaviour, ${ }^{14}$ attention problems $^{26}$ and somatic complaints. ${ }^{14}$

A careful interpretation of our findings is important, as the mean scores of both groups of children, for developmental outcome and behaviour problems, were within the normal range. A statistically significant difference of one point on the scaled score of the Bayley-III-NL, which was found for receptive communication skills after correcting age for prematurity, is not immediately clinically significant. Additionally, the effect sizes of the group differences were found to be small, except for uncorrected age scores. Group differences with medium to large effect sizes need immediate attention from professionals in order to try to reduce the developmental and behavioural problems found. Small sized group differences might, however, also be important, as these could be early indicators of later, more serious difficulties, having an effect on their functioning at school. Further study of development of these moderately preterm children from toddler age onwards is therefore needed to fully evaluate these findings.

The analyses in our study were adjusted for maternal education level. In our sample, more mothers of moderately preterm children had a low or medium education level than mothers of term born children. This is in accordance with population based studies showing that lower maternal education level is a risk factor for premature birth. ${ }^{27}{ }^{28}$ Correcting for maternal education level might therefore also lead to overcorrection. However, the number of low educated mothers was small in our moderately preterm and term born sample, which is a limitation of this study.

The relationship between neonatal characteristics and longterm developmental outcome measures was explored to investigate if indications for specific subgroups could be found. Comparison of late preterm born children (ie, GA 34-36 weeks) with the term born group showed the same results as for the total group of moderately preterm children: even late preterm children differed from term born children on several aspects of development and behaviour. With respect to other neonatal characteristics, no relationships were found between GA, birth weight, days in hospital, need for additional oxygen, and developmental and behavioural outcomes. As only a small percentage of the children had problems like hypoglycaemia (5.2\%) clearly indicated in their hospital discharge letters, our study had only limited power to find any differences between the subgroups of children with and without problems such as hypoglycaemia. 
Therefore, when the focus needs to be on such neonatal problems in moderately preterm children, future studies should be specifically designed to examine these problems.

A final remark is that in this study, only moderately preterm children who were relatively healthy and did not need tertiary NICU admittance, were selected. However, in the Netherlands $10.2 \%$ of all moderately preterm children are admitted to the NICU. ${ }^{18}$ This specific subgroup might be at an even higher risk of developmental and behavioural problems, and therefore these children should also be studied in the future.

In conclusion, at 2 years of age, uncorrected for prematurity, moderately preterm children differed from their term born peers in cognitive, motor and communication skills. With age corrected for prematurity, they differed from their term born peers only on receptive communication skills. Already at 2 years of age, moderately preterm children show more internalising behaviour problems than term born peers. Although only subtle differences were found in this study, these findings may still be an indication for later difficulties of moderately preterm children in academic functioning and socioemotional development.

Acknowledgements The authors thank all the parents and children for their participation. The study was conducted in cooperation with Meander Medical Centre (CBM) in Amersfoort, St. Antonius Hospital (J A Schipper) in Nieuwegein, Amstelland Hospital (L Spanjerberg) in Amstelveen, Flevo hospital (D Maingay) in Almere, Diakonessenhuis (I de Boer) in Utrecht, Hospital Gelderse Vallei (G van Enk, W Avis) in Ede, Tergooi Hospital (CAL) in Blaricum, Zuwe Hofpoort Hospital (E Visscher) in Woerden and University Obstetric Centre (I G M Berends and I Overbeeke) in Utrecht.

Contributors MdJ: designed the study, coordinated the data collection, carried out the analyses, drafted the initial manuscript and approved the final manuscript as submitted. MV: designed the study, reviewed and revised the manuscript and approved the final manuscript as submitted. CAL: assisted in data collection, reviewed the manuscript and approved the final manuscript as submitted. CBM: assisted in data collection, reviewed the manuscript and approved the final manuscript as submitted. ALvB: conceptualised and designed the study, reviewed and revised the manuscript and approved the final manuscript as submitted.

Patient consent Parental consent obtained.

Funding Utrecht University.

Competing interests None.

Ethics approval Medical ethical committee of the Utrecht Medical Center.

Provenance and peer review Not commissioned; externally peer reviewed.

\section{REFERENCES}

1 Blencowe $\mathrm{H}$, Cousens S, Oestergaard MZ, et al. National, regional, and worldwide estimates of preterm birth rates in the year 2010 with time trends since 1990 for selected countries: a systematic analysis and implications. Lancet 2012;379:2162-72.

2 Shapiro-Mendoza CK, Tomashek KM, Kotelchuck M, et al. Effect of late-preterm birth and maternal medical conditions on newborn morbidity risk. Pediatrics 2008; 121:e223-32

3 de Jong $M$, Verhoeven $M$, van Baar AL. School outcome, cognitive functioning, and behaviour problems in moderate and late preterm children and adults: a review. Semin Fetal Neonatal Med 2012;17:163-9.
4 Hillemeier MM, Farkas G, Morgan PL, et al. Disparities in the prevalence of cognitive delay: how early do they appear? Paediatr Perinat Epidemiol 2009;23:186-98

5 Morag I, Bart 0, Raz R, et al. Developmental characteristics of late preterm infants at six and twelve months: a prospective study. Infant Behav Dev 2013;36:451-6.

6 Nepomnyaschy L, Hegyi T, Ostfeld BM, et al. Developmental outcomes of late-preterm infants at 2 and 4 years. Matern Child Health J 2012;16:1612-24.

7 Romeo DM, Di Stefano A, Conversano M, et al. Neurodevelopmental outcome at 12 and 18 months in late preterm infants. Eur J Paediatr Neurol 2010;14:503-7.

8 Woythaler MA, McCormick MC, Smith VC. Late preterm infants have worse 24-month neurodevelopmental outcomes than term infants. Pediatrics 2011;127: e622-9.

9 Voigt B, Pietz J, Pauen S, et al. Cognitive development in very vs. moderately to late preterm and full-term children: Can effortful control account for group differences in toddlerhood? Early Hum Dev 2012;88:307-13.

10 Cheatham CL, Bauer PJ, Georgieff MK. Predicting individual differences in recall by infants born preterm and full term. Infancy 2006;10:17-42.

11 Darlow BA, Horwood LJ, Wynn-Williams MB, et al. Admissions of all gestations to a regional neonatal unit versus controls: 2-year outcome. J Paediatr Child Health 2009;45:187-93.

12 Blaggan S, Guy A, Boyle EM, et al. A parent questionnaire for developmental screening in infants born late and moderately preterm. Pediatrics 2014;134:e55-62.

13 Gray RF, Indurkhya A, McCormick MC. Prevalence, stability, and predictors of clinically significant behavior problems in low birth weight children at 3, 5, and 8 years of age. Pediatrics 2004;114:736-43.

14 Potijk MR, de Winter AF, Bos AF, et al. Higher rates of behavioural and emotional problems at preschool age in children born moderately preterm. Arch Dis Child 2012;97:112-17.

15 van Baar AL, Vermaas J, Knots $E$, et al. Functioning at school age of moderately preterm children born at 32 to 36 weeks' gestational age. Pediatrics 2009;124:251-7.

16 Achenbach TM, Rescorla L. ASEBA preschool forms \& profiles: an integrated system of multi-informant assessment. Aseba; 2000.

17 Stichting Perinatale Registratie Nederland. Referentiecurven. http://www.perinatreg nl/referentiecurven (accessed 25 Apr 2014).

18 Stichting Perinatale Registratie Nederland. Perinatale zorg in nederland 2012. Utrecht: Stichting Perinatale Registratie Nederland; 2013.

19 Steenis LJP, Verhoeven M, van Baar AL. The bayley III: the instrument for early detection of developmental delay. In: Columbus A, ed. Advances in psychology research. 92nd edn. Hauppauge, NY: Nova Science Publishers; 2012:133-41.

20 Van Baar AL, Steenis LJP, Verhoeven M, et al. Bayley Scales of Infant and Toddler Development-Derde Editie, Nederlandstalige bewerking, Technische handleiding. Amsterdam: Pearson Assessment and Information B.V., 2014.

21 Faul F, Erdfelder E, Lang A, et al. G* power 3: a flexible statistical power analysis program for the social, behavioral, and biomedical sciences. Behav Res Methods 2007;39:175-91

22 Cohen J. Statistical power for the social sciences. Hillsdale, NJ: Laurence Erlbaum and Associates, 1988.

23 Sugita $\mathrm{K}$, lai $\mathrm{M}$, Inoue $\mathrm{T}$, et al. Normative data and the effect of correction for prematurity on test scores in the psychomotor development of extremely low birthweight infants. Brain Dev 1990;12:334-8.

24 Siegel LS. Correction for prematurity and its consequences for the assessment of the very low birth weight infant. Child Dev 1983:1176-88.

25 Rickards A, Kitchen W, Doyle L, et al. Correction of developmental and intelligence test scores for premature birth. J Paediatr Child Health 1989;25:127-9.

26 Talge NM, Holzman C, Wang J, et al. Late-preterm birth and its association with cognitive and socioemotional outcomes at 6 years of age. Pediatrics 2010;126:1124-31.

27 Morgen CS, Bjork C, Andersen PK, et al. Socioeconomic position and the risk of preterm birth--a study within the Danish national birth cohort. Int J Epidemio 2008;37:1109-20.

28 Petersen CB, Mortensen LH, Morgen CS, et al. Socio-economic inequality in preterm birth: a comparative study of the nordic countries from 1981 to 2000. Paediatr Perinat Epidemiol 2009;23:66-75 